\title{
A COMMUTATIVITY THEOREM FOR SEMIPRIME RINGS
}

\author{
VISHNU GUPTA
}

(Received 23 February; revised 20 November 1979)

Communicated by D. E. Taylor

\begin{abstract}
It is shown that if $R$ is a semiprime ring with 1 satisfying the property that, for each $x, y \in R$, there exists a positive integer $n$ depending on $x$ and $y$ such that $(x y)^{k}-x^{k} y^{k}$ is central for $k=n, n+1, n+2$, then $R$ is commutative, thus generalizing a result of Kaya.
\end{abstract}

1980 Mathematics subject classification (Amer. Math. Soc.) : primary 16 A 70; secondary 16 A 12

Kaya (1976) showed that if $R$ is a primary ring (that is, $R / J(R)$ is simple) or semiprime ring with 1 satisfying the property that, for each $x, y \in R$, there exists a positive integer $n$ depending on $x$ and $y$ such that $(x y)^{k}=x^{k} y^{k}$ for $k=n, n+1, n+2$, then $R$ is commutative, thus generalizing a theorem of Luh (1971), p. 211, who proved the result for a fixed $n$ in the case when $R$ is primary. Ligh and Richoux (1977) has proved the result of Luh, for a fixed $n$, without assuming that $R$ is primary. Recently Richoux (to appear) has extended the Ligh-Richoux result to arbitrary $n$. In this note we prove the result stated in the abstract, which generalizes Theorem 2(ii) of Kaya (1976) for the semiprime ring case. However, it is not possible, by Example 2 of Luh (1971), to replace semiprime ring by primary ring in our result.

We use the following notations:

$$
\begin{aligned}
Z(R) & =\text { the centre of } R, \\
J(R) & =\text { the Jacobson radical of } R, \\
{[x, y] } & =x y-y x .
\end{aligned}
$$

For the sake of convenience, we label some properties of $R$ as follows.

(A) For each $x, y \in R$, there exists a positive integer $n$ depending on $x$ and $y$ such that $(x y)^{k}-x^{k} y^{k} \in Z(R)$ for $k=n, n+1, n+2$.

(B) For each $x, y \in R, x y+y x \in Z(R)$. 
(C) For each $x, y \in R$,

$$
y x^{2}+x^{2} y+y x^{2} y+2 y x y=x y^{2}+y^{2} x+x y^{2} x+2 x y x .
$$

LEMMA 1. If $R$ is a semisimple ring satisfying (A), then $R$ is commutative.

ProOF. The proof is based on standard technique given by Herstein (1961), p. 29 and Jacobson (1968), p. 220.

First we assume that $R$ is a division ring satisfying (A). Let $\left[(x y)^{k}-x^{k} y^{k}, z\right]=0$ for all $z \in R$. Replacing $z$ by $x y$ and $y x$, we get respectively,

$$
\left[x^{k-1} y^{k-1}, y x\right]=0
$$

and

$$
\left[(x y)^{k}-x^{k} y^{k}, y x\right]=0 .
$$

Let $k=n, n+1, n+2$. Then from (1) and (2) we get

$$
\begin{aligned}
{\left[(x y)^{n}, y x\right] } & =0, \\
{\left[(x y)^{n+1}, y x\right] } & =0 .
\end{aligned}
$$

The last two equations provide us with $x y^{2} x=y x^{2} y$. Now $R$ is commutative as a part of the proof of Theorem 2.5 of Gupta (1970).

Next we assume $R$ is a primitive ring satisfying (A). If $R$ is not a division ring, then $D_{2}$ the ring of $2 \times 2$ matrices over some division ring $D$ will be a homomorphic image of subring of $R$ and satisfies (A). But this is impossible as

$$
x=\left(\begin{array}{ll}
0 & 1 \\
0 & 0
\end{array}\right) \text { and } y=\left(\begin{array}{ll}
0 & 0 \\
1 & 0
\end{array}\right)
$$

fail to satisfy (A). Hence $R$ must be a division ring and therefore is commutative.

Finally if $R$ is semisimple ring satisfying (A), then $R$ is a subdirect sum of primitive rings $R_{\alpha}$ each of which, as a homomorphic image of $R$, satisfies (A) and hence is commutative by the above discussion. Thus $R$ is commutative.

We give the following lemma which will be used frequently in the subsequent study.

LEMMA 2. Let $R$ be a prime ring and $x \neq 0, y$ be elements of $R$. If $x$ and $x y$ are in $Z(R)$, then $y$ is in $Z(R)$.

PROOF. Let $x, x y$ be in $Z(R)$. Then $x y z=z x y=x z y$ for all $z \in R$. From this we have $x R(y z-z y)=0$. Since $R$ is a prime ring and $x \neq 0$ we get $z y=y z$ for all $z \in R$. Thus $y$ is in $Z(R)$. 
LemMA 3. If $R$ is a semiprime ring of characteristic 2 satisfying (B), then $R$ is commutative.

ProOF. Let us assume that $R$ is a prime ring satisfying (B). Replacing $x$ by $x y$ in (B), we get that $R$ is a commutative ring by an application of Lemma 2 .

If $R$ is a semiprime ring satisfying (B), then it is isomorphic to a subdirect sum of prime rings $R_{\alpha}$ each of which, as a homomorphic image of $R$, satifies (B) and hence is commutative by the above part. Thus $R$ is commutative.

LeMma 4. If $R$ is a semiprime ring satisfying $(C)$, then $R$ is commutative.

ProOF. It suffices to assume that $R$ is a prime ring, using a similar argument as given in the proof of Lemma 3. Replacing $x$ by $x+y$ in $(C)$ and cancelling using $(C)$, we get

$$
\left(y+y^{2}\right)[x, y]=[x, y]\left(y+y^{2}\right) .
$$

Replacing $x$ by $x y$ and $y x$ in (1), and adding the results we obtain

$$
\left(y+y^{2}\right)\left[x, y^{2}\right]=\left[x, y^{2}\right]\left(y+y^{2}\right) .
$$

Adding (5) and (6), we have

$$
\left(y+y^{2}\right)\left[x, y+y^{2}\right]=\left[x, y+y^{2}\right]\left(y+y^{2}\right)
$$

for all $x, y \in R$.

If the characteristic of $R$ is not 2, then by a sublemma of Herstein (1969), p. 5, we have

$$
y+y^{2} \in Z(R) \text { for all } y \in R .
$$

Replacing $y$ by $x+y$ in (8), we get

$$
x y+y x \in Z(R) \text { for all } x, y \in R .
$$

Replacing $x$ by $x y$ in (9) and by Lemma 2, we obtain $y \in Z(R)$ unless $x y+y x=0$ for every $x$. If $x y+y x=0$ for every $x$, then we replace $x$ by $y$ to get $2 y^{2}=0$, which will imply that $y^{2}=0$. By $(8) y \in Z(R)$ for all $y \in R$. Hence $R$ is commutative.

If the characteristic of $R$ is 2 , then by (7), we have

$$
y^{2}+y^{4} \in Z(R) \text { for all } y \in R .
$$

Replacing $y$ by $y^{2}$ in (10), we have

$$
y^{4}+y^{8} \in Z(R) \text { for all } y \in R .
$$

Adding (10) and (11), we obtain

$$
y^{2}+y^{8} \in Z(R) \text { for all } y \in R .
$$


Again replacing $y$ by $y^{3}$ in (10), we get

$$
\left(y^{2}+y^{8}\right) y^{4} \in Z(R) \text { for all } y \in R \text {. }
$$

By Lemma 2, $y^{4} \in Z(R)$ unless $y^{2}+y^{8}=0$. If $y^{4} \in Z(R)$, then by (10) $y^{2} \in Z(R)$. If $y^{2}+y^{8}=0$, then it can be seen that $y^{2}=0$ for all $y \in J(R)$. Hence in either case $y^{2} \in Z(J(R))$ for all $y \in J(R)$. Let $x \in J(R)$. Replacing $y$ by $x+y$, we get $x y+y x \in Z(J(R)) . J(R)$ is commutative by Lemma 3 .

Since $\bar{R}=R / J(R)$ is semisimple, it suffices to assume that $\bar{R}$ is a division ring, using a similar argument as given in the proof of Lemma 1 . By the argument of the above paragraph, we have $a^{2} \in Z(\bar{R})$ unless $a^{2}+a^{8}=0$. If $a^{2}+a^{8}=0$, then $a^{6}=1 \in Z(\bar{R})$. In either case $a^{6} \in Z(\bar{R})$ for all $a \in \bar{R} . \bar{R}$ is commutative by Lemma 1 of Belluce and others (1966). Now $J(R)$ is commutative and $x y-y x \in J(R)$ for all $x, y \in R$. By Lemma 1.5 of Herstein (1969) $x y-y x \in Z(R)$ for all $x, y \in R$. $R$ is commutative, again by Lemma 1.5 of Herstein (1969).

THEOREM 1. If $R$ is a semiprime ring with 1 satisfying (A), then $R$ is commutative.

Proof. Let $x, y \in J(R)$. Then $((1+x)(1+y))-(1+x)^{k}(1+y)^{k} \in Z(R)$, where $k=m, m+1, m+2$. Since $(1+x)$ and $(1+y)$ are invertible, we use the argument of Lemma 1 to obtain

$$
(1+x)(1+y)^{2}(1+x)=(1+y)(1+x)^{2}(1+y) .
$$

Thus $J(R)$ satisfies (C). By Lemma $4, J(R)$ is commutative. $R / J(R)$ is semisimple and satisfies (A), hence is commutative by Lemma 1 . Now $R$ is commutative as in the proof of Lemma 4.

The author is thankful to the referees for their helpful suggestions.

\section{References}

L. P. Belluce, I. N. Herstein and S. K. Jain (1966), 'Generalized commutative rings', Nagoya Math. J. 27, $1-5$.

R. N. Gupta (1970), 'Nilpotent matrices with invertible transpose', Proc. Amer. Math. Soc. 24, $572-575$.

I. N. Herstein (1961), 'Power maps in rings', Michigan Math. J. 8, 29-32.

I. N. Herstein (1969), Topics in ring theory (University of Chicago Press).

N. Jacobson (1968), Structure of rings (revised edition, Amer. Math. Soc. Colloq. Publ. 37, Amer. Math. Soc., Providence, R.I.).

A. Kaya (1976). 'On a commutativity theorem of Luh', Acta Math. Acad. Sci. Hungar. 28, 33-36.

S. Ligh and A. Richoux (1977), 'A commutativity theorem for rings', Bull. Austral. Math. Soc. 16, 75-77.

J. Luh (1971), 'A commutativity theorem for primary rings', Acta Math. Acad. Sci. Hungar. 22, $211-213$.

P.O. Box 13261

Department of Mathematics

Alfateh University, Tripoli

Libya 